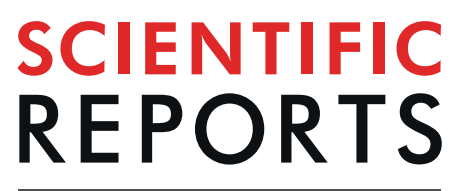

natureresearch

\title{
Diagnostic performance of a vessel- length-based method to compute the instantaneous wave-free ratio in coronary arteries
}

Kyung Eun Lee ${ }^{1,2}$, Gook Tae Kim ${ }^{1}$, Eui Cheol Jung ${ }^{1}$, Eun Seok Shin $\mathbb{1 0}^{3,4^{*}}$ \& Eun Bo Shim ${ }^{1,4^{*}}$

The instantaneous wave-free ratio (iFR) is a recently introduced vasodilator-free index to assess the functional severity of coronary stenosis in the resting state, while fractional flow reserve (FFR) is the gold standard index in hyperemia. The computed instantaneous wave-free ratio (CT-iFR) is a noninvasive method to estimate iFR using computer simulations. Here, we developed a vessel-lengthbased CT-iFR method in patient-specific models of coronary arteries. This method was implemented by coupling a three-dimensional computational fluid dynamics model with a lumped parameter model (LPM) of coronary circulation in a non-hyperemic resting state. A time-varying resistance in the LPM was used for the iFR simulation. In total, 50 coronary vessels of 32 patients were computed, and their CT-iFR values were compared with clinically measured iFRs to evaluate the diagnostic performance of the present CT-iFR method. The area under the receiver operating characteristics curve of CT-iFR validation was 0.93 . In diagnostic performances of CT-iFR, accuracy, sensitivity, and specificity were $86 \%, 83.3 \%$, and $86.8 \%$, respectively. These results indicate that this CT-iFR method can be used as a pre-operative aid to establish a percutaneous coronary intervention strategy as a noninvasive alternative to iFR.

Fractional flow reserve (FFR) is widely used to evaluate the functional severity of stenosis in coronary arteries. This index is the ratio of the distal pressure $\left(\mathrm{P}_{\mathrm{d}}\right)$ to the proximal pressure $\left(\mathrm{P}_{\mathrm{a}}\right)$ through a coronary stenosis under vasodilator (adenosine)-induced hyperemia ${ }^{1-3}$. Here, the pressures are the averaged values over the whole cardiac cycle. On the other hand, the instantaneous wave-free ratio (iFR) is the flow reserve under non-hyperemic resting conditions, thus in the absence of vasodilators ${ }^{4-6}$. In the $i F R$, the ratio of $\mathrm{P}_{\mathrm{d}} / \mathrm{P}_{\mathrm{a}}$ is measured during a wave-free period, ranging from mid- to late-diastole, when microvascular resistance is naturally constant and minimized in the cardiac cycle ${ }^{4-6}$. The diagnostic performances of iFR and FFR to determine the need for percutaneous coronary intervention (PCI) have been investigated in many clinical studies ${ }^{7-11}$. These clinical studies have reported that iFR is as accurate as FFR in estimating the hemodynamic severity of coronary stenosis. A recent comparative clinical study of iFR- versus FFR-guided strategies in 2,492 and 2,037 patients revealed that coronary revascularization therapy guided by iFR is comparable to that by FFR, with respect to the risk of major adverse cardiac events ${ }^{12-14}$.

Taylor et al. ${ }^{15}$ pioneered non-invasive estimation using computed FFR (CT-FFR; the prefix CT- indicates 'computed') in the three-dimensional (3D) geometry of patient-specific coronary arteries reconstructed from computed tomography (CT) images and validated the clinical diagnostic performance and utility of the method in several studies ${ }^{16-18}$. Recently, we also proposed a vessel-length-based CT-FFR method ${ }^{19-21}$ and presented the clinical diagnostic performance of the method $^{22}$. Similar to the CT-FFR approach, computed iFR (CT-iFR) provides the means to noninvasively calculate iFR values in a patient-specific $3 \mathrm{D}$ geometry of the coronary arteries. Indeed, the CT-iFR method using commercial software, ANSYS (Canonsburg, USA), was proposed in an existing study ${ }^{23}$; however, the clinical efficacy and diagnostic performance of the method were not fully investigated in the

${ }^{1}$ Department of Mechanical and Biomedical Engineering, Kangwon National University, Chuncheon, Kangwon-do, 200-701, Republic of Korea. ${ }^{2}$ Bio-Convergence Technology Group, Korea Institute of Industrial Technology, Jeju, 63243, Republic of Korea. ${ }^{3}$ Division of Cardiology, Department of Internal Medicine, Ulsan University Hospital, University of Ulsan College of Medicine, Dong-gu, Ulsan, 682-714, Republic of Korea. ${ }^{4}$ These authors contributed equally: Eun Seok Shin and Eun Bo Shim. *email: sesim1989@gmail.com; ebshim@kangwon.ac.kr 


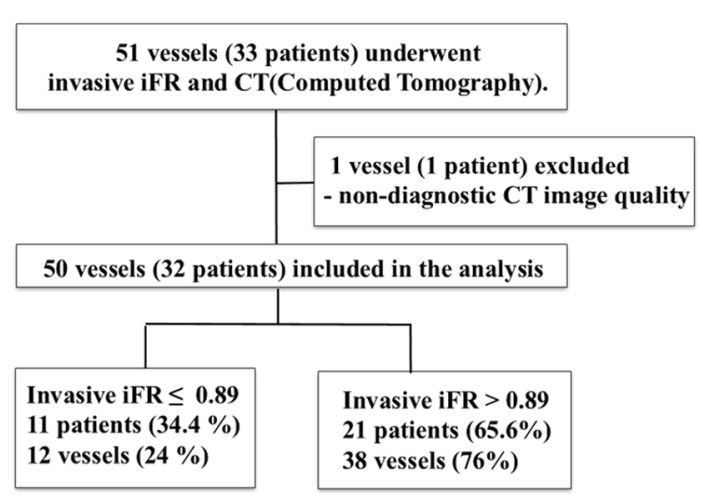

(a)

\begin{tabular}{lll}
\hline Characteristics & Median & Q1-Q3 \\
\hline Age & 57.0 & $51.5-61.0$ \\
Height $(\mathrm{cm})$ & 169.0 & $164.0-173.0$ \\
Weight $(\mathrm{kg})$ & 69.0 & $64.0-77.0$ \\
$\begin{array}{l}\text { Systolic blood pressure } \\
\text { SBP (mmHg) }\end{array}$ & 124.5 & $117.5-142.0$ \\
$\begin{array}{l}\text { Diastolic blood pressure } \\
\text { DBP(mmHg) }\end{array}$ & 77.0 & $71.0-85.5$ \\
$\begin{array}{l}\text { Heart rate } \\
\text { HR (bpm) }\end{array}$ & 72.5 & $65.5-78.5$ \\
$\begin{array}{l}\text { Hematocrit } \\
\text { HCT (\%) }\end{array}$ & 42.3 & $40.6-44.6$ \\
$\begin{array}{l}\text { Stroke Volume } \\
\text { SV(ml) }\end{array}$ & 64.0 & $49.5-76.5$ \\
\hline \hline
\end{tabular}

(b)

Figure 1. (a) Study enrollments for 51 vessels of 33 patients, (1 vessel of 1 patient was excluded). Fifty vessels in 32 patients were included in the study. (b) Clinical characteristics of the 32 patients. Clinical data are expressed as medians and interquartile ranges (Q1-Q3). (Unit: bpm, beats per min).

research. To our knowledge, there has been no reported study addressing the clinical validity or performance of the CT-iFR method by comparing CT-iFR values with clinically measured iFRs (M-iFR; the prefix M- indicates 'pressure-wire measurement').

In this study, we address the clinical validity and performance of the CT-iFR method. For this purpose, a simulation method to noninvasively estimate iFR values in the $3 \mathrm{D}$ geometry of coronary arteries is proposed. This method is implemented by extending the vessel-length-based CT-FFR approach presented in our previous reports $^{20,21}$, in which a computational fluid dynamics (CFD) model of coronary arteries is coupled with a lumped parameter model (LPM) to reflect the effect of coronary microvascular and venous hemodynamics. Here, the parameters of LPM are determined based on the vessel lengths of coronary arteries. To investigate the clinical efficacy and performance of the present CT-iFR method, we simulated the coronary arterial hemodynamics of 32 patients and calculated their iFR values. The simulated CT-iFR values were compared with clinically measured iFR values (M-iFR). Finally, the clinical benefits and limitations of the CT-iFR method are discussed.

\section{Results}

A clinically measured iFR (M-iFR). In total, 51 vessels of 33 patients underwent coronary CT angiography and invasive iFR (M-iFR), as presented in Fig. 1(a). One patient was excluded due to the poor diagnostic quality of a blurred CT image; thus, a total of 50 vessels of 32 patients were simulated in this study. Figure 1(a) presents the prevalence of the clinically measured $\mathrm{M}$-iFR values to show the distribution of positive and negative vessel samples. The numbers of vessels of the left anterior descending artery (LAD), left circumflex artery (LCX), and right coronary artery (RCA) in 32 patients were 28 (56\%), 15 (30\%), and 7 (14\%), respectively. Figure 1(b) shows the patients' physiological characteristics (age, height, weight, blood pressure, heart rate, hematocrit, and stroke volume) with the medians and interquartile ranges (Q1-Q3). Among the 50 vessels included in the study, 38 vessels $(76 \%)$ were negatively diagnosed (iFR $>0.89$ ), while the number of positively diagnosed vessels (iFR $\leq 0.89$ ) was 12 (24\%). Vessels had a stenosis area-based stenosis degree (\%) of $72 \pm 13$ (mean \pm standard deviation). Calcifications were observed in 2 vessels (of two patients) among the 50 vessels. The number of positively diagnosed vessels (stenosis degree $>75 \%$ ) was 22 using CT angiography area-based diagnosis, whereas the number of positively diagnosed vessels (iFR $\leq 0.89)$ was $12(24 \%)$ using an iFR-based diagnosis.

Computed iFR (CT-iFR). CT-iFR (the pressure ratio across the stenosis over the wave-free period under non-hyperemic resting conditions) was calculated by a multi-scale simulation technique, as described in our previous papers ${ }^{20,21}$. The method couples the 3D local CFD of patient-specific coronary arterial hemodynamics with the vessel-length-based LPM of coronary microvascular and venous hemodynamics. Figure 2 shows the clinically measured iFR and the CT-iFR for a representative case; Fig. 2(a) shows the M-iFR value at the measured point of iFR marked with a red arrow on the X-ray angiography image, and Fig. 2(b) shows the computed CT-iFR distribution in the patient-specific $3 \mathrm{D}$ coronary model. The clinically measured iFR value and the computed iFR showed good agreement. A reduction in the CT-iFR value was observed in the very distal part of the iFR-measured vessel (LAD), due to arterial curvature and arterial tapering close to capillaries (Fig. 2).

Comparisons between clinically measured iFR (M-iFR) and computed iFR (CT-iFR). We compared computed CT-iFR results with clinical M-iFR values on a per-vessel basis $(n=50)$. Figure 3 shows a scatter plot and Bland-Altman plot of the clinical data (M-iFR) and the computed results (CT-iFR) in panels (a) and (b), respectively. Computed CT-iFR results showed good agreement with clinical M-iFR data $(n=50)$ in Fig. 3(a). We also observed good correlation between CT-iFR and M-iFR values (Pearson's correlation coefficient, $\left.\rho_{p}=0.85, \mathrm{P}<0.0001\right)$. Figure $3(\mathrm{~b})$ presents the Bland-Altman plot, with a mean difference of 0.01 and a $95 \%$ limit 


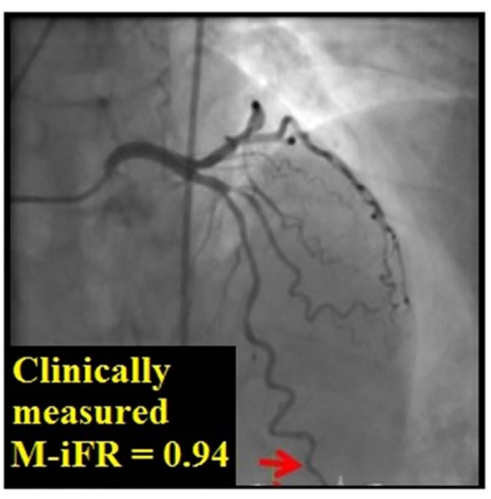

(a)

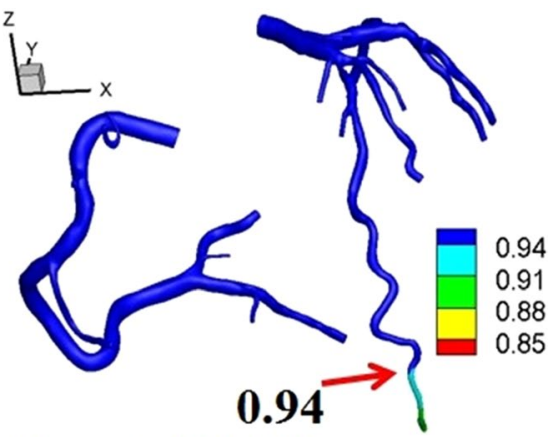

Computed CT-iFR

(b)

Figure 2. (a) Clinically measured instantaneous wave-free ratio (iFR) data (M-iFR) and (b) computed iFR results (CT-iFR) in a representative case (Case 1). Here, prefixes M- and CT- refer to 'clinically measured' data and 'computed' results, respectively.

of agreement over the range -0.13 to $0.14(n=50)$. A slight underestimation of CT-iFR relative to M-iFR was evident, as shown in Fig. 3(b).

Evaluation of diagnosis performances. Figure 4 presents the three representative cases of iFR distribution, including the patient-specific vessel-length ratio. In the classification of four groups using the terms of true/ false and positive/negative, true or false refers to the assigned classification being correct or incorrect; positive or negative refers to the presence or absence of disease. Figure 4(a) shows a representative case of a true negative group, in which a patient of M-iFR $>0.89$ was identified correctly as a patient of CT-iFR $>0.89$. Figure 4(b) shows a representative case of a true positive group in which a patient of $\mathrm{M}$-iFR $\leq 0.89$ was classified correctly as a patient of CT-iFR $\leq 0.89$. There was good diagnostic agreement between $\mathrm{M}$-iFR and CT-iFR readings, as shown in Fig. 4(a,b). Figure 4(c) shows a representative case of a false negative group, in which a patient of M-iFR $\leq 0.89$ was identified incorrectly as a patient of CT-iFR $>0.89$. Notably, there was diagnostic disagreement between M-iFR and CT-iFR, despite small differences in the values between M-iFR and CT-iFR [Fig. 4(c)]. Figure 4(c) shows the case in which a sick patient was identified as a healthy person. In the case of such intermediate stenosis, small differences can lead to incorrect plans calling for stenting or deference of treatment. A more accurate, non-invasive iFR approach would help to establish better strategies for intermediate stenosis.

The diagnostic performance of CT-iFR was evaluated using receiver operating characteristics (ROC) curve analysis. Figure $3(\mathrm{c})$ shows the ROC curve of CT-iFR using a cut-off clinical iFR of $\leq 0.89(n=50)$. The area under the ROC curve (AUC) value for the diagnostic performance of CT-iFR was 0.933 ( $95 \%$ confidence interval (CI): 0.868-0.998). Diagnostic performances of CT-iFR in terms of the accuracy, sensitivity, and specificity are shown in Fig. 3(c), labeled as ACC, SN, and SP, respectively, in the figure. Prevalence and accuracy were $24 \%$ and 86\%, respectively. Sensitivity and specificity were $83.3 \%$ (95\% CI: 51.5-97.9\%) and 86.8\% (95\% CI: 71.9-95.6\%), respectively. Positive and negative likelihood ratios were 6.33 (95\% CI: 2.7-14.9) and 0.19 (95\% CI: 0.05-0.7), respectively. In Table 1, the diagnostic performance of the present CT-iFR method is compared with that of CT-FFR reported in the literature. All data are from studies of per-vessel basis. One of the CT-FFR studies in Table 1 was written by our group (Chung et al. ${ }^{22}$ ); the others used the method of HeartFlow, Inc. ${ }^{16-18,24}$ (Redwood City, CA, USA). The number of vessels in the present CT-iFR study was 50, which was somewhat less than those of other studies, with the exception of two ${ }^{23,24}$. Among the clinical validation studies of CT-FFR shown in Table 1, the NXT study showed better clinical performance than the other studies. The clinical performance of our current CT-iFR study approached that of the NXT study in terms of sensitivity, specificity, accuracy, and AUC. Moreover, in terms of Pearson's correlation coefficient, the present CT-iFR study $(0.85)$ showed more accurate results than the NXT study (0.82).

\section{Discussion}

FFR is a gold standard for PCI strategy and is measured over an entire cardiac cycle under a hyperemic state induced by adenosine infusion. Recently, another physiological index, iFR, was proposed for clinical PCI strategy. Unlike FFR, iFR is measured during a wave-free period of the cardiac cycle under non-hyperemic resting conditions ${ }^{4}$. Recent clinical investigations published in the New England Journal of Medicine reported that iFR is comparable to FFR, in terms of diagnostic performance in PCI strategy ${ }^{12,13}$. Now, a variety of clinical studies to delineate the diagnostic characteristics of the iFR index are currently underway. Along with invasive measurement of iFR, a noninvasive computational approach, CT-iFR, has been proposed. CT-iFR is a very powerful diagnostic technique for specific patients, for example, those without hemodynamic response to intravenous adenosine, as well as for patients with ventricular hypertrophy and other conditions. Notably, the resting state is significantly associated with clinical outcome. However, there has been no clinical validation study of CT-iFR. Considering that CT-FFR was used in the PCI strategy after several clinical validation studies ${ }^{16-18}$, evaluation of the diagnostic performance of CT-iFR is a prerequisite for its clinical application. 


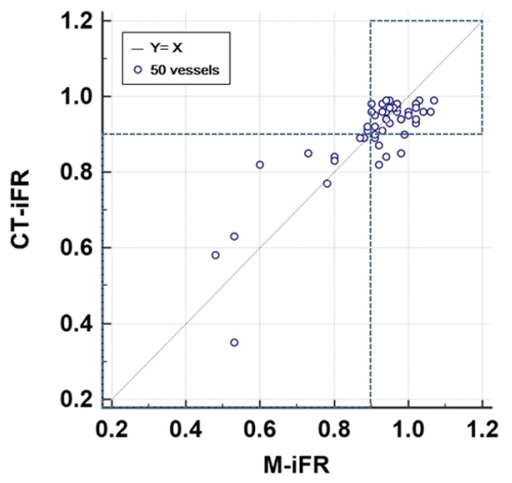

(a)

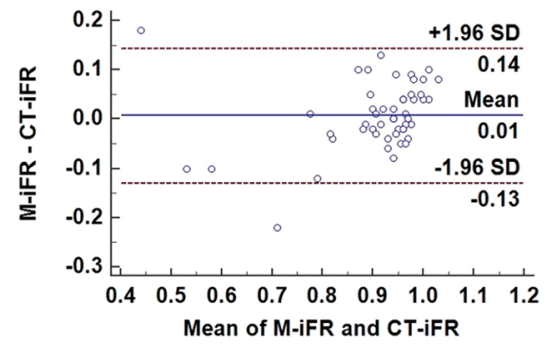

(b)

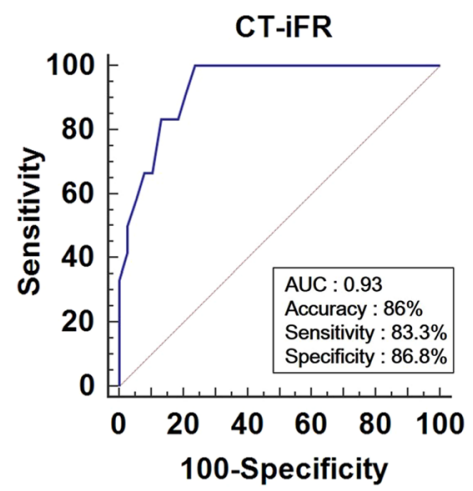

(c)

Figure 3. Scatter plot and Bland-Altman plot on a per-vessel basis. (a) Scatter plot of iFR between clinical M-iFR data and computed CT-iFR results. (b) Bland-Altman plot of iFR between clinical M-iFR data and computed CT-iFR results. (c) Receiver-operating characteristic (ROC) curve of CT-iFR using the cut-off M-iFR of $\leq 0.89$ on a per-vessel basis.

There are two main contributions of the present study. First, we propose a vessel-length-based CT-iFR method. This method is similar to the CT-FFR method ${ }^{20,21}$ applied in our earlier study. In the CT-iFR method of the current study, a patient-specific 3D CFD model is combined with the vessel-length-based LPM of coronary circulation. Under hyperemic conditions for the CT-FFR calculation, the microvascular resistance of the LPM is a constant value over the entire cardiac cycle, as shown in Fig. 5(h). However, in the CT-iFR method, the resistance is a time-varying value under non-hyperemic resting conditions, as shown in Fig. 5(h). As far as we know, there has been no microvascular resistance model that takes into account such time-varying characteristics. To resolve this problem, we propose a simplified time-varying trapezoidal resistance method, mimicking existing clinical observations $\mathrm{s}^{25}$ [Fig. 5(g)]; this method was tested to determine its validity via clinical outcome verification.

Second, we found that the clinical diagnostic performance of CT-iFR was comparable to that of CT-FFR. Here, CT-iFR simulations were performed for 50 vessels in 32 patients for comparisons with clinically measured M-iFR data (Table 1). Of the 32 patients, 11 patients tested positive $(\mathrm{M}-\mathrm{iFR} \leq 0.89)$. The number of positive vessel samples was 12 among the 50 vessels in Fig. 1(a). CT-iFR simulations showed a diagnostic performance with an AUC of 0.93 on a per-vessel basis (Fig. 3), indicating good diagnostic performance in CT-iFR computations. Among the validation studies of computed FFR (CT-FFR) in Table 1, the NXT study ${ }^{18}$ showed better clinical performance over the criteria specified, with the exception of sensitivity, compared with the other studies. The clinical performance of the present CT-iFR method almost equalled that of the NXT study in terms of sensitivity, specificity, and accuracy. Moreover, Pearson's correlation coefficient of the present CT-iFR study was 0.85 , indicating more accurate results than the coefficient determined in the NXT study of 0.82 . However, in terms of sensitivity, the present CT-iFR method was inferior to CT-FFR methods, although the sensitivity of the DeFACTO study was lower.

Our study had several other limitations. First, the number of patients with measured iFRs was relatively small. As shown in Table 1, the present CT-iFR study had a lower vessel number than most of the CT-FFR studies, except for the studies of Ma et al. ${ }^{23}$ and Kim et al. ${ }^{24}$. The computed iFR obtained by the current method showed better diagnostic performance than the previously computed iFR by Ma et al.; however, it would be difficult to compare the diagnostic performance with the previous one $e^{23}$, as both studies were performed in relatively small study populations and for selected population samples. Therefore, our future work will include a larger number of blood vessels to validate the CT-iFR approach. Second, the patient-specific time-varying resistance of the microvascular model is not perfect. An observed microvascular resistance pattern in 51 patients in the previous study ${ }^{25}$ was used for the time-varying resistance pattern of this study, regardless of the patients' specific condition. However, variations in patients' conditions may need to be considered in certain cases (e.g., multi-vessel disease, diffuse disease, advanced micro-vessel circulation disease, etc.); thus, this is a limitation of this study. For multi-vessel diseases, increasing the population of this patient group is necessary. However, the numbers for this patient group do not tend to reach the required enrollment quickly; thus, the study would have to be conducted over a longer term. For diffuse diseases, the mean stenosis length and mean stenosis degree in diffuse stenosis are important factors in understanding the effects on the microcirculatory system; these two factors are also necessary for clinical studies. Influences on the microcirculatory system can be studied by grouping patients according to the stenosis length and the stenosis degree. However, long-term investigations should be planned for this. Third, identifying the correct stenosis severity and correct lesion length can be difficult in calcified vessels. This is because calcification produces image noise, and the calcified area can be misinterpreted as arterial lumen. In this study, two calcified vessels were included. The area of lumen was overestimated; thus, the CT-iFR reading was overestimated compared with that using M-iFR. Calcification may degrade the performance of noninvasive iFR, particularly for patients of an advanced age. In this study, a small number of calcified vessels was included. Increasing the number of calcified vessels may increase the misdiagnosis of stenosis severity; however, the limited number of calcified vessels was also a limitation of the current study. Fourth, we did not use a turbulent model (i.e., a k- $\varepsilon$ model); nevertheless, turbulent effects may not be negligible in vessels with severe stenosis. The absence of a turbulence 
a) $\mathrm{M}$-iFR $>0.89 \&$ CT-iFR $>0.89$

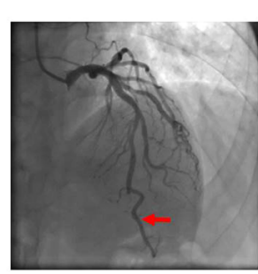

M-iFR $=0.95$

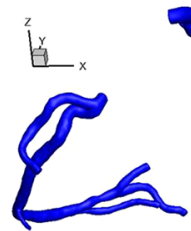

(1)

CT-iFR $=0.99$

Length ratio

RCA : LAD : LCX = 355.5mm : 398.6mm : 286.4mm

b) M-iFR $\leq 0.89 \&$ CT-iFR $\leq 0.89$

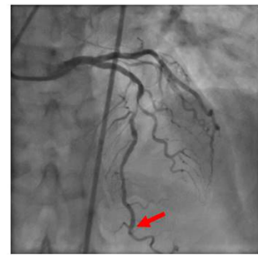

$\mathrm{M}-\mathrm{iFR}=\mathbf{0 . 7 8}$

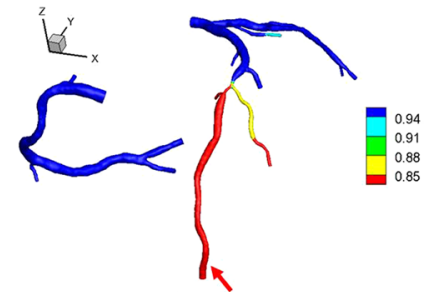

CT-iFR $=0.77$

Length ratio

RCA : LAD : LCX = 353.5mm : 323.9mm : 228.5mm

c) $\mathrm{M}-\mathrm{iFR} \leq 0.89 \& \mathrm{CT}$-iFR $>0.89$

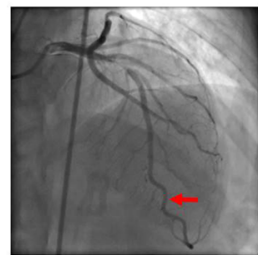

M-iFR $=0.89$

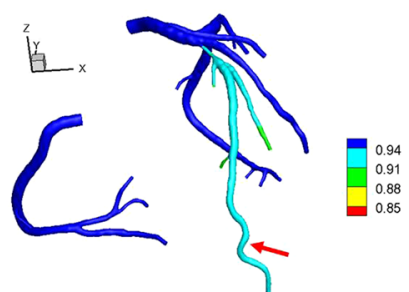

CT-iFR $=0.92$

Length ratio

RCA : LAD : LCX $=269.6 \mathrm{~mm}: 375.4 \mathrm{~mm}: 364.6 \mathrm{~mm}$

Figure 4. Three representative cases of iFR distribution, including a patient-specific vessel-length ratio, where (a) M-iFR $>0.89$ and CT-iFR $>0.89$, (b) M-iFR $\leq 0.89$ and CT-iFR $\leq 0.89$, and (c) M-iFR $\leq 0.89$ and CTiFR $>0.89$.

\begin{tabular}{|l|l|l|l|l|l|l|l|}
\hline Study name & Reference & $\begin{array}{l}\text { No. of } \\
\text { vessels }\end{array}$ & Sensitivity & Specificity & Accuracy & $\begin{array}{l}\text { Area under } \\
\text { curve (AUC) }\end{array}$ & $\begin{array}{l}\text { Pearson's correlation } \\
\text { coefficient }\end{array}$ \\
\hline DISCOVER-FLOW (CT-FFR) & Koo et al. ${ }^{16}$ & 159 & $88 \%$ & $82 \%$ & $84 \%$ & 0.90 & 0.72 \\
\hline DeFACTO (CT-FFR) & Min et al. ${ }^{17}$ & 407 & $80 \%$ & $63 \%$ & $69 \%$ & 0.81 & 0.63 \\
\hline NXT (CT-FFR) & Norgaard et al. ${ }^{18}$ & 484 & $84 \%$ & $87 \%$ & $86 \%$ & 0.93 & 0.82 \\
\hline CT-FFR study & Kim et al. ${ }^{24}$ & 48 & $85 \%$ & $57 \%$ & $77 \%$ & - & 0.60 \\
\hline NOVEL-FLOW & Chung et al..$^{22}$ & 218 & $86 \%$ & $86 \%$ & $85 \%$ & 0.93 & 0.76 \\
\hline Previous other CT-iFR study & Ma et al. ${ }^{23}$ & 47 & 70.6 & 83.3 & 78.7 & 0.87 \\
\hline Present CT-iFR study & & 50 & 83.3 & 86.8 & $86 \%$ & 0.93 \\
\hline
\end{tabular}

Table 1. Diagnostic performance comparison of the present CT-iFR method with CT-FFR performance from published works ${ }^{16-18,22-24}$. 
model in this study may have led to overestimated CT-iFR values, compared with clinical iFR measurements. Therefore, the iFR value might be lowered further and might show better diagnostic performance if the turbulence effect were taken into account. Lastly, there are difficulties in clarifying the causes of error in individual CT-iFR calculations. Errors can arise from many sources in CT-iFR. For example, segmentation errors derived from images due to image resolution or a calcified vessel, etc.; errors derived from the vessel-length method; errors derived from neglecting advanced microcirculatory disease; and 4) errors derived from neglecting the turbulence effect in severe stenosis cases. Thus, given the potential error from all of these sources, it is not simple to estimate the error separately for individual vessels. We attempted to determine the error on a case by case basis.

We do not consider that these limitations significantly influenced our major findings. To our knowledge, this is the first report in which CT-iFR values were compared with clinically measured iFR data (M-iFR) for clinical validation. The diagnostic performance evaluation in this study shows that CT-iFR can be used as a pre-operative aid to establish PCI strategy as a noninvasive alternative to M-iFR.

\section{Methods}

Study population and clinical measurements. In total, 51 vessels of 33 patients underwent coronary $\mathrm{CT}$ angiography and invasive iFR; however, 1 patient was excluded due to the poor diagnostic quality of a blurred CT image [Fig. 1(a)]. Therefore, we used a sample set of 50 vessels from 32 patients who underwent coronary CT angiography examinations and iFR measurements at Ulsan University Hospital (Ulsan City, Republic of Korea) (Fig. 1). The inclusion criteria were: patients who were at least 18 years old and suspected of having coronary artery disease, and who had $>30 \%$ stenosis by visual estimation in major epicardial coronary arteries and underwent successful iFR measurement. Coronary X-ray angiography, coronary CT angiography, and iFR examinations followed current guidelines ${ }^{1,2,25,26}$. For the coronary CT, we used a dual-source CT scanner platform (Definition CT, Siemens, Forchheim, Germany; Aquilion ONE, Toshiba, Otawara, Japan). The scanning parameters were: tube voltage, $100 \mathrm{kV}$; tube current, $320 \mathrm{~mA}$; CT slice thickness, $0.6 \mathrm{~mm}$; and number of pixels, $512 \times 512$. Scans were performed with prospective electrocardiogram triggering. The numbers of coronary CT slices obtained ranged from 300 to 600 slices. For CT acquisition, sublingual nitroglycerin was given, and $\beta$-blockers were administered to patients with a rapid heart rate, according to the Society of Cardiovascular Computed Tomography (SCCT) guidelines ${ }^{26}$. Contrast agent was injected, followed by a saline flush. CT was scanned for all coronary arteries, including the left ventricle and proximal ascending aorta. iFR measurements were also made according to current guidelines ${ }^{26}$. Cardiac catheterization was performed via a femoral approach. At the start of the procedure, unfractionated heparin (5000 IU) was given intravenously. A 0.014-inch pressure-sensor-tipped wire (PrimeWire Prestige; Volcano Corporation, San Diego, CA, USA) was positioned at the tip of a guiding catheter. After intracoronary administration of nitrate and pressure equalization at the tip of the catheter, the wire was advanced into the target vessel as distally as reasonably possible for pressure recordings. Physiological data for iFR were calculated automatically online using a commercially available system (S5 Imaging System; Volcano Corporation). iFR, the ratio of the mean distal coronary pressure to the mean proximal pressure during the wavefree period, was obtained using a catheter guide-wire (see Fig. 1 of refs. 4, 6, and 25$)^{4,6,25}$. An iFR value $\leq 0.89$ is considered the cut-off value to indicate significant myocardial ischemia ${ }^{27}$.

Numerical models and methodology. The main procedures of this study to compute CT-iFR are shown in Fig. 5. This method was originally proposed in our previous paper ${ }^{19}$ in 2014 and has been validated clinically in our subsequent studies ${ }^{20,21}$. More details were explained in the previous studies ${ }^{19-22}$. A non-invasive computer simulation method to evaluate the iFR value is based on patients' CT images and the physiological data. The method uses a CFD approach for the hemodynamics of the coronary arteries coupled with the LPM of the coronary circulation system, as in our previous papers ${ }^{20-22}$. To calculate the LPM parameters, such as resistances and capacitances, we employed the novel vessel-length-based method ${ }^{21}$. We measured the vascular centerline length from CT images. Then, we first obtained each summed length of the LAD, LCX, and RCA. A detailed explanation is given in our previous papers ${ }^{20-22}$. In the equations, resistance, denoted as ' $\mathrm{R}$ ', is inversely proportional to vessel length, $l$, indicating that a longer vessel length induces less resistance and thus more blood flow. Here, $\alpha$ is set to 3.45 , reducing the effect of the RCA vessel length feeding the right ventricle (RV) muscle on RCA flow. ' $k$ ' is a proportionality constant derived from the pressure-flow rate relation, as explained in our previous paper ${ }^{21}$ :

$$
\begin{gathered}
R_{L A D}=\frac{k}{l_{L A D}}, \\
R_{L C X}=\frac{k}{l_{L C X}}, \\
R_{R C A}=\frac{\alpha k}{\left(l_{R C A}\right)_{R V}}+\frac{k}{\left(l_{R C A}\right)_{L V}}, \\
R_{L A D}=\frac{k}{l_{L A D}}, R_{L C X}=\frac{k}{l_{L C X}}, R_{R C A}=\frac{\alpha k}{\left(l_{R C A}\right)_{R V}}+\frac{k}{\left(l_{R C A}\right)_{L V}},
\end{gathered}
$$



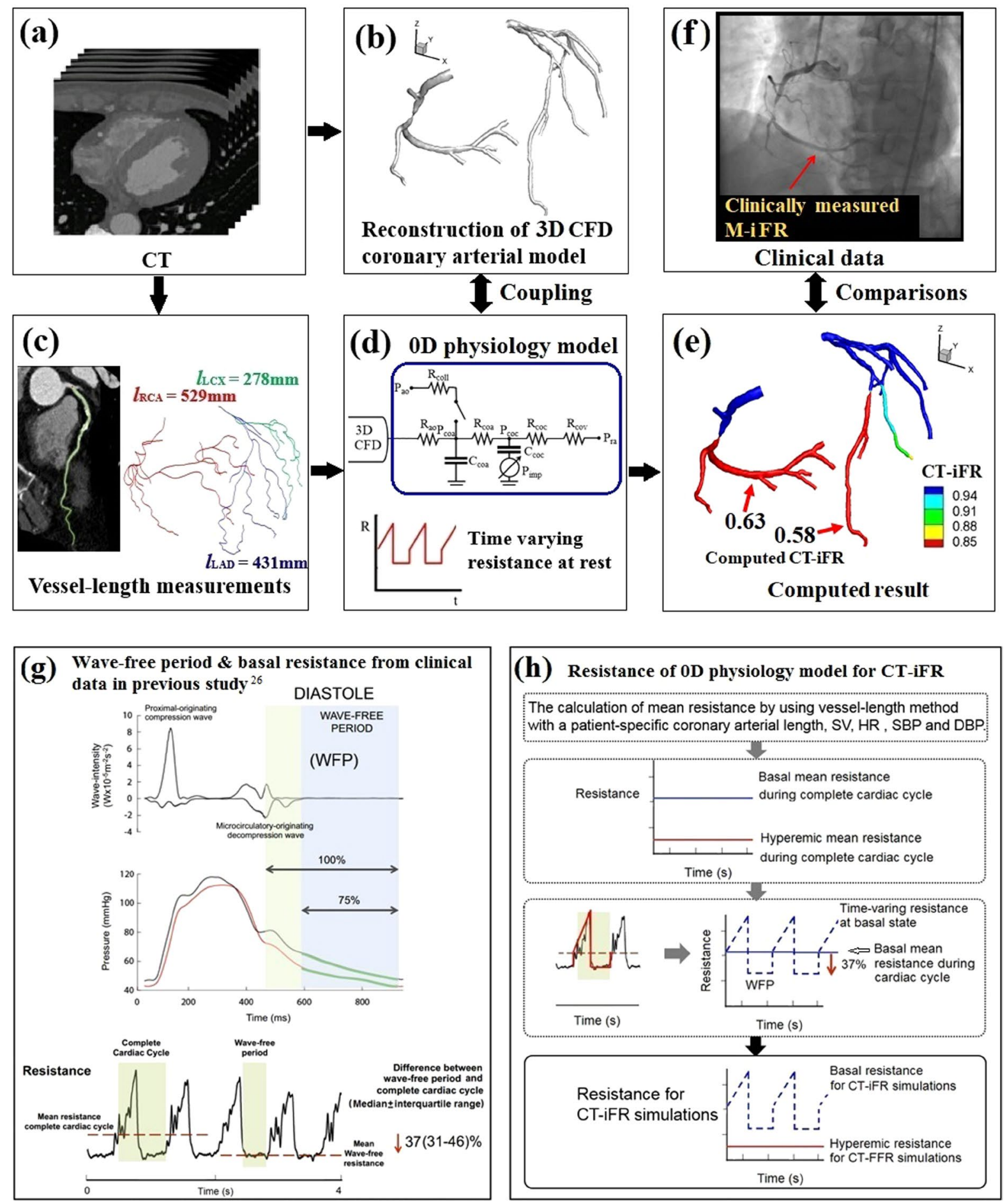

Figure 5. Procedure of computer simulation to compute CT-iFR. (a) Patient-specific coronary computed tomography (CT) angiogram acquisition. (b) Reconstruction of three-dimensional (3D) computational fluid dynamics (CFD) coronary model from CT images. (c) Coronary vessel length measurement from CT, where $l$ is the vessel length of 'LCX,' 'LAD,' and 'RCA, referring to the left circumflex artery, left anterior descending artery, and right coronary artery, respectively. (d) Lumped parameter model (LPM) establishment using a vessel-length based method. LPM consists of pressure 'P', resistance 'R', and capacitance 'C' elements. The subscripts for 'P, 'R', and 'C' are 'ao,' 'coll,' 'coa,' 'coc', 'cov', and 'ra', indicating aorta, collateral circulation, coronary arterial compartment, coronary capillary compartment, coronary venous compartment, and right atrium, respectively. Basal timevarying resistance was used for CT-iFR simulations. (e) Computed CT-iFR results obtained by coupling between the 3D CFD model and the LPM. (f) Clinical validation by comparing the computed iFR results with the clinically measured iFR data at the identical location. (g) Wave-free period, pressure waveform, and time-varying resistance pattern from clinical data of a previous paper ${ }^{25}$ (these figures are reproduced from Figs. 1 and 7 of ref. ${ }^{25}$ ). (h) Nonhyperemic time-varying resistance establishment for the LPM of CT-iFR by approximating trapezoidal patterns. 


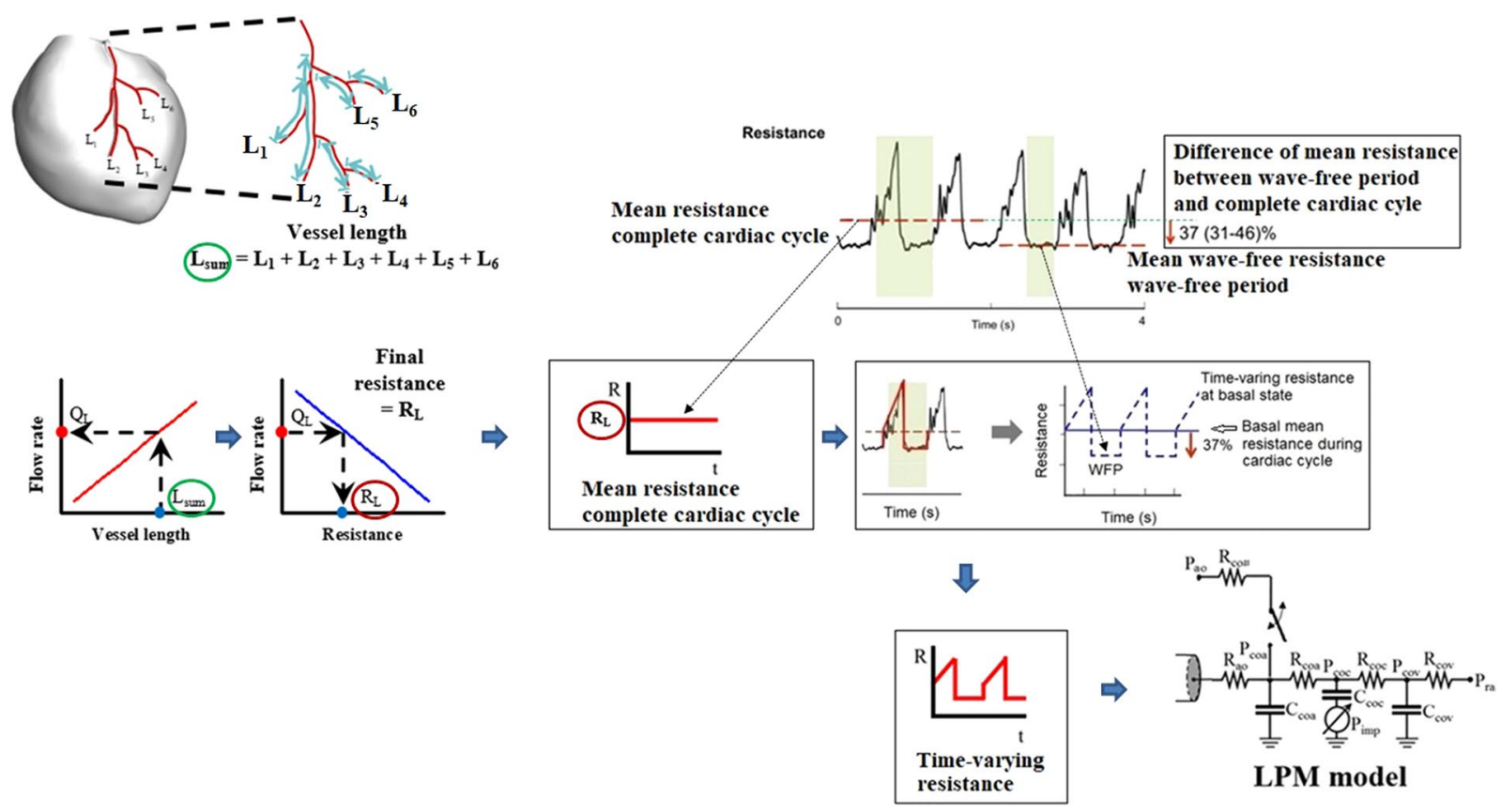

Figure 6. Schematic diagram of the vessel length method to estimate microvascular resistance of a coronary vessel at rest. The inset of the resistance plot (right upside panel) was copied from ref. ${ }^{25}$.

Criterion diameter of vessel : $1 \mathrm{~mm}$

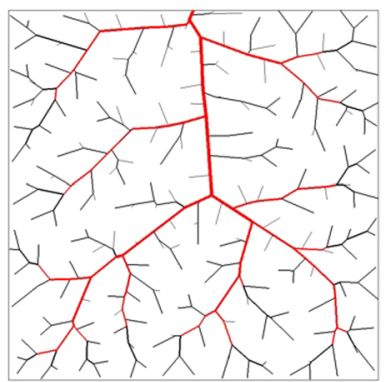

Area : $0.09 \mathrm{~m}^{2}$

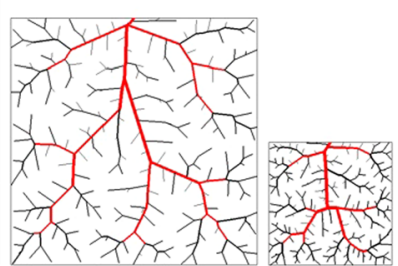

Area : $0.04 \mathrm{~m}^{2}$

Area : $0.01 \mathrm{~m}^{2}$

Vascular tree with 200 terminal segments

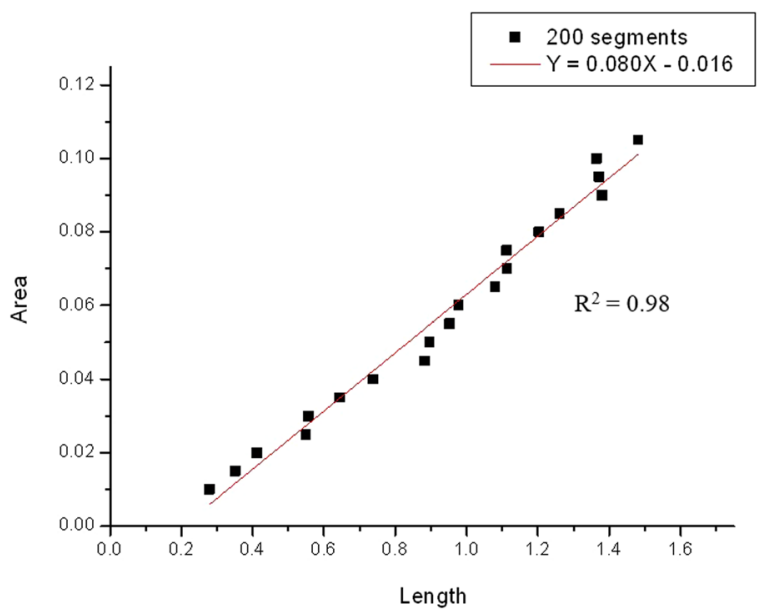

Figure 7. Optimized vascular fractal tree modeling in various areas (e.g., $0.09 \mathrm{~m}^{2}, 0.04 \mathrm{~m}^{2}$, and $0.01 \mathrm{~m}^{2}$ ) (left panel), and the linear relationship between the area (volume) and the total length of the blood vessel (right panel).

$$
\begin{gathered}
Q_{\text {total }}=C O * 0.04 \quad(C O: \text { cardiac output }) \\
=Q_{L A D}+Q_{L C X}+Q_{R C A}, \\
Q_{\text {total }}=\frac{\left(P_{\text {aorta }}-P_{\text {vein }}\right)}{R_{L A D}}+\frac{\left(P_{\text {aorta }}-P_{\text {vein }}\right)}{R_{L C X}}+\frac{\left(P_{\text {aorta }}-P_{\text {vein }}\right)}{R_{R C A}} .
\end{gathered}
$$

In Eq. (6), $P_{\text {aorta }}$ is calculated from the measured blood pressure of the patient, and $P_{\text {vein }}$ is assumed to be $2 \mathrm{mmHg}$. $Q_{\text {total }}$ is calculated from the cardiac output $(C O)$, which is based on the measured heart rate and stroke volume. If we substitute $R_{L A D}, R_{L C X}$, and $R_{R C A}$ of Eq. (6) with the values obtained from Eq. (4), then we can obtain the value of ' $k$ ', a patient-specific proportionality constant.

A schematic diagram of the present vessel-length method to estimate LPM resistance at rest is shown in Fig. 6. The LPM for the coronary circulation is used to represent the coronary vasculature from the small-size coronary arteries to the right atrium (Figs. 5 and 6). In the LPM, the coronary circulation involves three compartments: the coronary arteries (subscript coa), capillaries (subscript coc), and veins (subscript cov) [Fig. 5(d)]. The effect of 


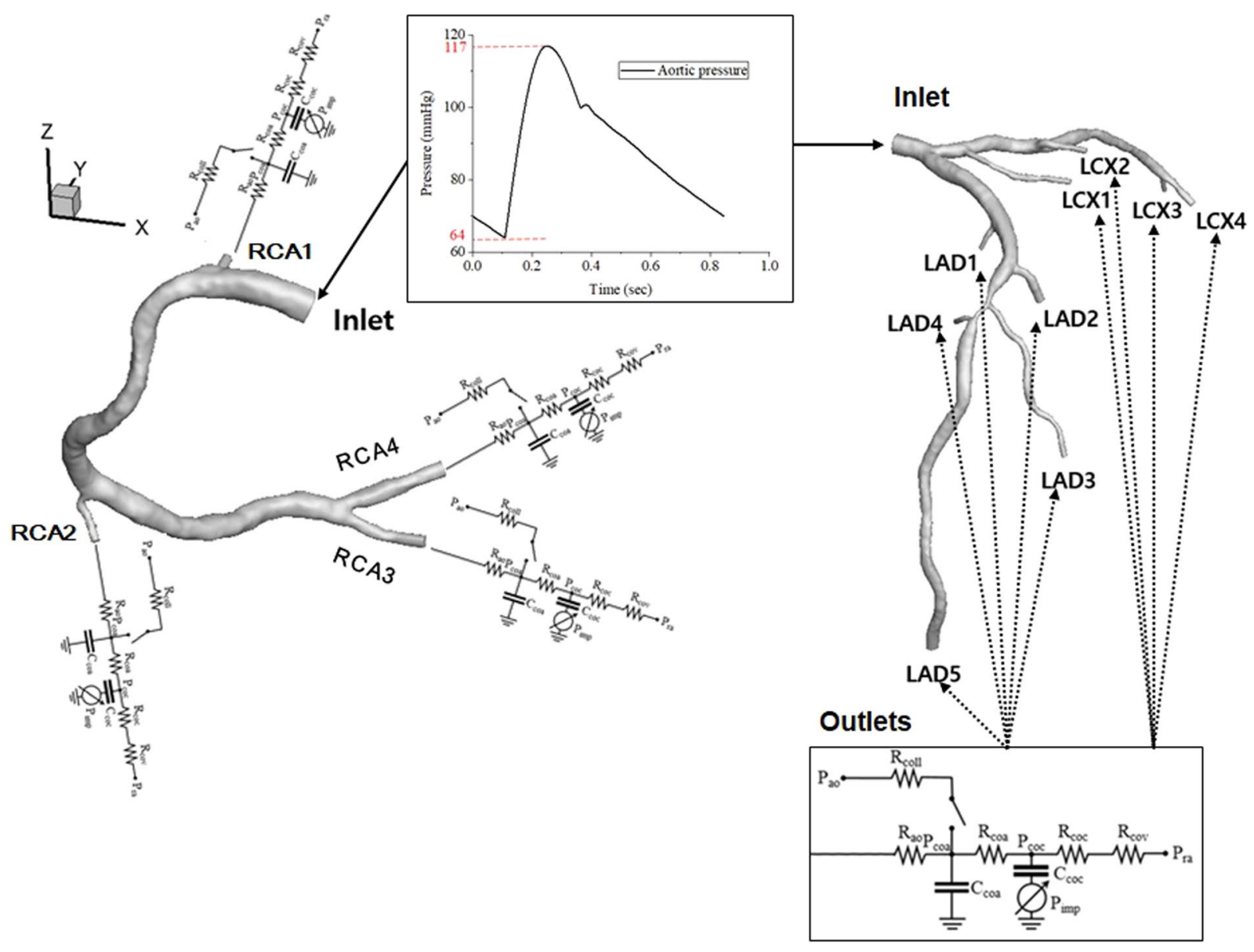

Figure 8. Representative 3D patient-specific CFD model, including inlet and outlet conditions (parameters in Tables 2-4).

myocardial squeezing by ventricular muscle contraction is approximated by the intra-myocardial pressure (subscript imp), and collateral flow (subscript coll) is considered using the switching system. The model is formulated in terms of an electric analog model consisting of elements such as resistors, capacitors, and a diode.

To reconstruct the geometry of the 3D CFD model, CT image slices were segmented using semi-automated commercial software (Aquarius, ver. 4.4.11; TeraRecon, Inc., Foster City, CA, USA). We reconstructed the vessel based on the identification of the vessels in CT images by a lumen cross-sectional area of $1.5 \mathrm{~mm}^{2}$. We applied this value to all vessels, given that there is a linear relationship between the area (volume) and the total length of a blood vessel (the right graph of Fig. 7), when creating a network of small arterial blood vessels that supply blood flow to a certain area (or volume) (left panel of Fig. 7). The figure is based on an optimized vascular fractal tree modeling technique ${ }^{28}$ and the length of the artery above a certain diameter, as shown in red (the left panel in Fig. 7). From this correlation we can observe important physiological principles. In a particular artery branch, measuring the total length of blood vessels belonging to that branch can infer the total area (or volume) of tissue covered by the branch. However, its volume is directly proportional to the blood flow that the branch feeds. Thus, this information can be used to determine the endovascular resistance of that branch. For mesh generation of the reconstructed 3D model, a spatial resolution (mesh size for the $3 \mathrm{D}$ model) was approximated as $0.0001 \mathrm{~m}$, and the number of elements was estimated to be in the range of $3 \times 10^{6}$ to $2 \times 10^{7}$. The temporal resolution (time resolution of the time steps) was $0.002 \mathrm{~s}$.

Numerical simulations of blood flow behavior in the 3D CFD model were performed using a Fortran-based in-house code, an incompressible Navier-Stokes solver, based on a segregated finite-element method ${ }^{19}$. The inlet boundary conditions for the simulation were estimated from the aortic pressure from clinically measured blood pressure and the heart rate from a patient's brachial artery ${ }^{20,21}$. Briefly, local hemodynamics in a 3D CFD model were coupled with global hemodynamics in the vessel-length-based LPM by an iterative method to update the pressure and flow rate ${ }^{19}$. In the iterative method, in each time step the computed flow rate in the outlet of the 3D CFD model was transferred to the LPM. We calculated the pressure and the flow rates in the LPM by solving the ordinary differential equations. The computed pressure in the LPM was used for the outlet condition of the 3D CFD model.

More details of these patient-specific parameters used in the CFD are presented using a representative case (Figs. 8 and 9, and Tables 2-4) that included the ratio of the length of the RCA, LAD, LCX, blood pressure, stroke volume, heart rate, hematocrit ratio, etc. Figure 8 shows a representative case of a 3D patient-specific CFD model 

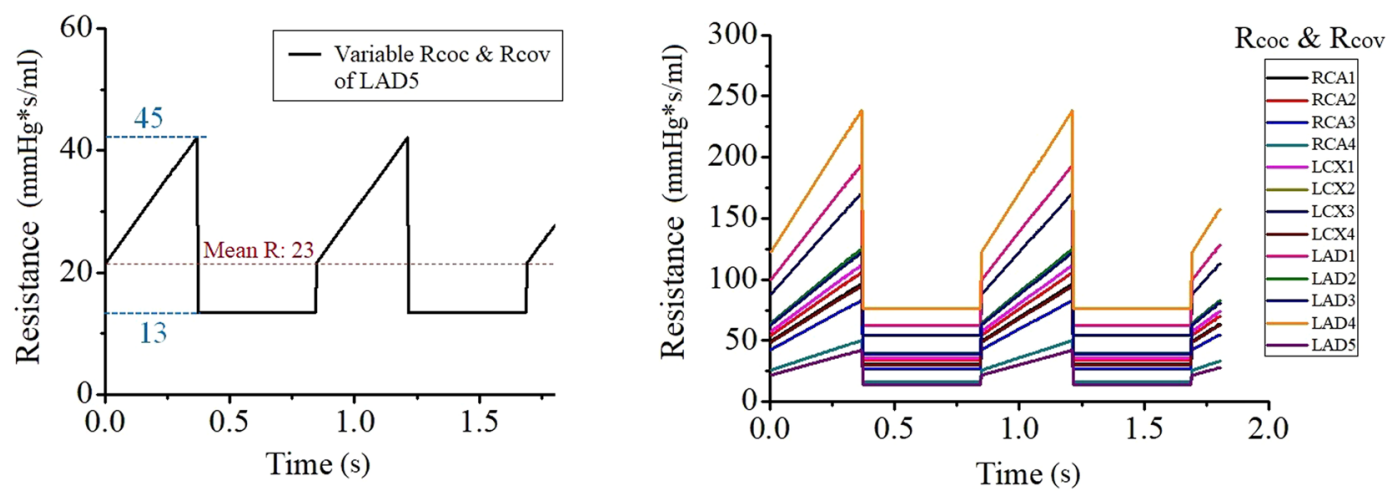

Figure 9. Time-varying Rcoc and Rcov [resistances for capillaries (subscript coc) and veins (subscript cov)] patterns in the model of Fig. 8. Time-variable Rcoc and Rcov patterns of LAD 5 (left panel). Time variable Rcoc and Rcov patterns of each branch (right panel) (Unit: R, mmHg.s/mL; C, $\mathrm{mL} / \mathrm{mmHg}$ ).

\begin{tabular}{|l|l|l|l|l|}
\hline SBP(mmHg) & DBP(mmHg) & HR & Stroke volume(ml) & Hematocrit(\%) \\
\hline 117 & 64 & 71 & 67 & 40.1 \\
\hline
\end{tabular}

Table 2. Patient-specific physiological parameters in the coronary model of Fig. 8. HR: heart rate.

\begin{tabular}{|l|l|l|l|}
\hline Artery & RCA & LAD & LCX \\
\hline Length $(\mathrm{mm})$ & 353.5 & 323.9 & 228.5 \\
\hline
\end{tabular}

Table 3. Patient-specific length ratio of RCA, LAD, and LCX in the coronary model of Fig. 8. RCA: right coronary artery; LAD: left anterior descending artery; LCX: left circumflex artery.

\begin{tabular}{|l|l|l|l|l|l|}
\hline & Mean of $\mathbf{R}_{\mathrm{ao}}$ & Mean of $\mathbf{R}_{\text {coa }}$ & Mean of $\mathbf{R}_{\text {coc }}$ \& $\mathbf{R}_{\text {cov }}$ & Mean of $\mathbf{C}_{\text {coa }}$ & Mean of $\mathbf{C}_{\text {coc }}$ \\
\hline RCA1 & 98.074 & 159.370 & 49.037 & 0.000312 & 0.005932 \\
\hline RCA2 & 107.464 & 174.629 & 53.732 & 0.000285 & 0.005414 \\
\hline RCA3 & 84.283 & 136.960 & 42.141 & 0.000363 & 0.006903 \\
\hline RCA4 & 51.009 & 82.890 & 25.505 & 0.000600 & 0.011406 \\
\hline LCX1 & 113.691 & 184.747 & 56.845 & 0.000269 & 0.005117 \\
\hline LCX2 & 125.138 & 203.349 & 62.569 & 0.000245 & 0.004649 \\
\hline LCX3 & 173.631 & 282.150 & 86.815 & 0.000176 & 0.003351 \\
\hline LCX4 & 96.146 & 156.237 & 48.073 & 0.000318 & 0.006051 \\
\hline LAD1 & 197.201 & 320.452 & 98.601 & 0.000155 & 0.002950 \\
\hline LAD2 & 127.300 & 206.863 & 63.650 & 0.000241 & 0.004570 \\
\hline LAD3 & 124.130 & 201.712 & 62.065 & 0.000247 & 0.004687 \\
\hline LAD4 & 242.554 & 394.150 & 121.277 & 0.000126 & 0.002399 \\
\hline LAD5 & 46.283 & 75.210 & 23.141 & 0.000662 & 0.012570 \\
\hline
\end{tabular}

Table 4. Lumped parameter model in the coronary model of Fig. 8 (the mean values of vessel-specific resistances $(\mathrm{R})$ and capacitances $(\mathrm{C})$ (units: $\mathrm{R}, \mathrm{mmHg} \cdot \mathrm{s} / \mathrm{mL} ; \mathrm{C}, \mathrm{mL} / \mathrm{mmHg}$ ).

coupling the inlet and outlet conditions. Table 2 shows patient-specific physiological parameters and Table 3 shows the patient-specific length ratio of RCA, LAD, and LCX. Table 4 presents the LPM of Fig. 8 that includes the mean values of vessel-specific resistances $(\mathrm{R})$ and capacitances $(\mathrm{C})$ (units: $\mathrm{R}, \mathrm{mmHg} \cdot \mathrm{s} / \mathrm{mL}, \mathrm{C}, \mathrm{mL} / \mathrm{mmHg}$ ). Figure 9 shows the time variable Rcoc and Rcov [resistances for capillaries (subscript coc) and vein (subscript cov)] patterns in the model of Fig. 8. The left panel shows the time-variable Rcoc and Rcov patterns of LAD 5, and the right panel shows the time-variable Rcoc and Rcov patterns of each branch. The patient-specific proportionality constant, $\mathrm{k}$, was 21673 for the model shown in Fig. 8. The spatial resolution (mesh size for the 3D model) was approximately $0.0002 \mathrm{~m}$, and the number of elements was approximately $8.6 \times 10^{5}$. The temporal resolution (time resolution of the time step) was $0.002 \mathrm{~s}$. Figure 4 (b) displays the CT-iFR results in the coronary model of Fig. 8. A more detailed explanation of the coupling method between the 3D CFD model and the LPM can be found in our previous papers ${ }^{19-21}$. 
Statistical analyses. Comparisons were made between clinical data (M-iFR) and computed values (CT-iFR) for validation. All data are expressed as the median and the interquartile range (Q1-Q3). We analyzed data on a per-vessel basis using commercial software (OriginPro 8, Northampton, MA, USA, and MedCalc, Mariakerke, Belgium). In a per-vessel basis analysis, 50 measured M-iFR data and computed CT-iFR values were included. The extent of the agreement between computed and clinical indices was evaluated by drawing Bland-Altman plots and scatter plots. We plotted ROC curves for CT-iFR, assuming an iFR cut-off value of 0.89 as the reference standard. The performance of CT-iFR is shown in terms of AUC, sensitivity and specificity with $95 \%$ confidence intervals.

Received: 16 August 2018; Accepted: 20 December 2019;

Published online: 24 January 2020

\section{References}

1. Pijls, N. H. et al. Fractional flow reserve. A useful index to evaluate the influence of an epicardial coronary stenosis on myocardial blood flow. Circulation. 92, 3183-3193 (1995).

2. Pijls, N. H. et al. Measurement of fractional flow reserve to assess the functional severity of coronary-artery stenosis. J. Med. 334, 1703-1708 (1996).

3. Pijls, N. H. \& De Bruyne, B. Coronary pressure. Second edition. 1-448 (Springer, 2000).

4. Sen, S. et al. Development and validation of a new adenosine-independent index of stenosis severity from coronary wave-intensity analysis: results of the ADVISE (ADenosine Vasodilator Independent Stenosis Evaluation) study. J. Am Coll Cardiol. 59, 1392-1402 (2012).

5. Escande, J. et al. Prospective assessment of the diagnostic accuracy of instantaneous wave-free ratio to assess coronary stenosis relevance: Results of ADVISE II International, Multicenter Study (ADenosine Vasodilator Independent Stenosis Evaluation II). JACC Cardiovasc Interv. 8, 824-833 (2015)

6. Berry, C. et al. VERIFY (VERification of Instantaneous Wave-Free Ratio and Fractional Flow Reserve for the Assessment of Coronary Artery Stenosis Severity in EverydaY Practice): a multicenter study in consecutive patients. J. Am Coll Cardiol. 61, $1421-1427$ (2013).

7. Jeremias, A. et al. Multicenter core laboratory comparison of the instantaneous wave-free ratio and resting $\mathrm{Pd} / \mathrm{Pa}$ with fractional flow reserve: the RESOLVE study. J. Am Coll Cardiol. 63, 1253-1261 (2014).

8. Park, J. J. et al. Clinical validation of the resting pressure parameters in the assessment of functionally significant coronary stenosis; results of an independent, blinded comparison with fractional flow reserve. Int J. Cardiol. 168, 4070-4075 (2013).

9. Petraco, R. et al. Real-time use of instantaneous wave-free ratio: results of the ADVISE in-practice: an international, multicenter evaluation of instantaneous wave-free ratio in clinical practice. Am Heart J. 168, 739-748 (2014).

10. Petraco, R. et al. Classification performance of instantaneous wave-free ratio (iFR) and fractional flow reserve in a clinical population of intermediate coronary stenoses: results of the ADVISE registry. EuroIntervention. 9, 91-101 (2013).

11. Nijjer, S. S. et al. Improvement in coronary haemodynamics after percutaneous coronary intervention: assessment using instantaneous wave-free ratio. Heart. 99, 1740-1748 (2013).

12. Davies, J. E. et al. Use of the instantaneous wave-free ratio or fractional flow reserve in PCI. N. Engl. J. Med. 376, 1824-1834 (2017).

13. Götberg, M. et al. Instantaneous Wave-Free Ratio versus Fractional Flow Reserve to guide PCI. N. Engl. J. Med. 376, 1813-1823 (2017).

14. Götberg, M. et al. Instantaneous Wave-Free Ratio versus Fractional Flow Reserve guided intervention (iFR-SWEDEHEART): Rationale and design of a multicenter, prospective, registry-based randomized clinical trial. Am Heart J. 170, 945-950 (2017).

15. Taylor, C. A., Fonte, T. A. \& Min, J. K. Computational fluid dynamics applied to cardiac computed tomography for noninvasive quantification of fractional flow reserve. J. Am Coll Cardiol. 61, 2233-2241 (2013).

16. Koo, B. K. et al. Diagnosis of ischemia-causing coronary stenoses by noninvasive fractional flow reserve computed from coronary computed tomographic angiograms: results from the prospective multicenter DISCOVER-FLOW (Diagnosis of Ischemia-Causing Stenoses Obtained Via Noninvasive Fractional Flow Reserve) study. J. Am Coll Cardiol. 58, 1989-1997 (2011).

17. Min, J. K. et al. Diagnostic accuracy of fractional flow reserve from anatomic CT angiography. JAMA. 308, 1237-1245 (2012).

18. Nørgaard, B. L. et al. Diagnostic performance of noninvasive fractional flow reserve derived from coronary computed tomography angiography in suspected coronary artery disease: the NXT trial (Analysis of Coronary Blood Flow Using CT Angiography: Next Steps). J. Am Coll Cardiol. 63, 1145-1155 (2014).

19. Kwon, S. S. et al. A novel patient-specific model to compute coronary fractional flow reserve. Prog Biophys. Mol. Biol. 116, 48-55 (2014).

20. Lee, K. E. et al. A patient-specific virtual stenotic model of the coronary artery to analyze the relationship between fractional flow reserve and wall shear stress. Int J. Cardiol. 222, 799-805 (2016).

21. Lee, K. E. et al. Estimation of the flow resistances exerted in coronary arteries using a vessel length-based method. Pflugers Arch. 468, 1449-1485 (2016).

22. Chung, J. H. et al. Diagnostic performance of a novel method for fractional flow reserve computed from noninvasive computed tomography angiography (NOVEL-FLOW Study). Am J. Cardiol., https://doi.org/10.1016/j.amjcard.2017.04.057. (2017).

23. Ma, Y. et al. Instantaneous wave-free ratio derived from coronary computed tomography angiography in evaluation of ischemiacausing coronary stenosis. Medicine. 96, e5979 (2017).

24. Kim, K. H. et al. A novel noninvasive technology for treatment planning using virtual coronary stenting and computed tomographyderived computed fractional flow reserve. JACC Cardiovasc Interv. 7, 72-78 (2014).

25. Sen, S. et al. Diagnostic classification of the instantaneous wave-free ratio is equivalent to fractional flow reserve and is not improved with adenosine administration. Results of CLARIFY (Classification Accuracy of Pressure-Only Ratios Against Indices Using Flow Study). J. Am Coll Cardiol. 61, 1409-1420 (2013).

26. Abbara, S. et al. SCCT guidelines for performance of coronary computed tomographic angiography: a report of the Society of Cardiovascular Computed Tomography Guidelines Committee. J. Cardiovasc Comput Tomogr. 3, 190-204 (2009).

27. Petraco, R. et al. Hybrid iFR-FFR decision-making strategy: implications for enhancing universal adoption of physiology-guided coronary revascularization. Eurolntervention. 8, 1157-1165 (2013).

28. Schreiner, W. Computer generation of complex arterial tree models. J. Biomed. Eng. 15, 148-150 (1993).

\section{Acknowledgements}

This research was supported by the National Research Foundation of Korea (NRF) grant (NRF2018R1D1A1B07044528) and Kangwon National University (Proejct No: D1001177-01-01). For Figures 5 and 6, we appreciate for permissions of the reproduction of figures 1 and 7 in ref. 25. 


\section{Author contributions}

Lee K.E. provided the main idea for this research. Lee K.E. and Shim E.B. wrote the main manuscript text. Kim G.T. and Jung E.C. assisted in statistical analyses. Shin E.S. provided the clinically measured data and medical advice. All authors reviewed this manuscript. Shim E.B. and Shin E.S. contributed equally as corresponding authors.

\section{Competing interests}

The authors declare no competing interests.

\section{Additional information}

Correspondence and requests for materials should be addressed to E.S.S. or E.B.S.

Reprints and permissions information is available at www.nature.com/reprints.

Publisher's note Springer Nature remains neutral with regard to jurisdictional claims in published maps and institutional affiliations.

(c) (1) Open Access This article is licensed under a Creative Commons Attribution 4.0 International License, which permits use, sharing, adaptation, distribution and reproduction in any medium or format, as long as you give appropriate credit to the original author(s) and the source, provide a link to the Creative Commons license, and indicate if changes were made. The images or other third party material in this article are included in the article's Creative Commons license, unless indicated otherwise in a credit line to the material. If material is not included in the article's Creative Commons license and your intended use is not permitted by statutory regulation or exceeds the permitted use, you will need to obtain permission directly from the copyright holder. To view a copy of this license, visit http://creativecommons.org/licenses/by/4.0/.

(C) The Author(s) 2020 